\title{
Optimizing radionuclide imaging in the assessment of cardiac sarcoidosis
}

\author{
John P. Bois, $M D{ }^{a}$ and Panithaya Chareonthaitawee, $M^{a}$ \\ a Division of Cardiovascular Diseases, Mayo Clinic, Rochester, MN
}

Received Jul 21, 2015; accepted Jul 21, 2015

doi:10.1007/s12350-015-0252-y

\section{See related article, pp. 244-52}

Sarcoidosis is a disease of unknown etiology and is characterized by the development of non-caseating granulomas with a predilection for the pulmonary system. Involvement of the cardiovascular system ranges from $20 \%$ in the United States to over $75 \%$ in Japan. ${ }^{1}$ Up to $85 \%$ of sarcoid-related mortality results from cardiac sarcoidosis (CS). ${ }^{2}$ Observational studies suggest that early initiation of glucocorticoids may inhibit the inflammatory response to $\mathrm{CS}$, thereby limiting fibrotic formation within the heart and ultimately improving long-term survival. ${ }^{3}$ Consequently, prompt and accurate diagnosis of CS is critical given its prognostic and therapeutic ramifications. Unfortunately, the diagnosis of CS has proven challenging since approximately half the of patients with CS are initially asymptomatic ${ }^{1}$ and, due to heterogenous myocardial involvement, CS detection by endomyocardial biopsy (EMB) has a sensitivity of only $20 \%$ to $30 \% .{ }^{4}$ Gallium $\left({ }^{67} \mathrm{Ga}\right)$ singlephoton emission tomography (SPECT) is specific for CS but has a sensitivity of less than $40 \% .^{5}$ Non-radionuclide techniques for the diagnosis of CS include echocardiography which has a poor sensitivity $(25 \%)^{4}$ and cardiac MRI which has substantially greater sensitivity for CS detection at $75 \%{ }^{6}$ but may be contraindicated in some patients with suspected CS such as those with cardiac devices.

Cardiac imaging with fluorine-18 fluorodeoxyglucose $\left({ }^{18} \mathrm{FDG}\right)$ positron emission tomography (PET) appears to have high diagnostic accuracy for CS as

Reprint requests: Panithaya Chareonthaitawee, MD, Division of Cardiovascular Diseases, Mayo Clinic, 200 First Street SW, Rochester, MN 55905; chareonthaitawee.panithaya@mayo.edu

J Nucl Cardiol 2016;23:253-5.

$1071-3581 / \$ 34.00$

Copyright (C) 2015 American Society of Nuclear Cardiology. demonstrated in several observational studies. ${ }^{7}$ Consequently, experts have recently included cardiac ${ }^{18}$ FDGPET imaging in the standard diagnostic algorithm for $\mathrm{CS}^{8}$ The gradual adoption of $\operatorname{cardiac}{ }^{18}$ FDG-PET imaging as a standard of care in the assessment and treatment of CS underscores the need for studies focusing on standardization of patient preparation, imaging protocols, and interpretation of cardiac ${ }^{18}$ FDGPET imaging for CS.

An important component of cardiac imaging with ${ }^{18}$ FDG-PET is patient preparation. The objective of patient preparation for an ${ }^{18}$ FDG-PET CS imaging protocol is to suppress physiologic myocardial ${ }^{18} \mathrm{FDG}$ uptake in order to enhance detection of pathologic ${ }^{18}$ FDG uptake in inflamed tissues. ${ }^{9}$ Under postprandial conditions, increased serum insulin levels upregulate glucose transporter 1 and 2 (GLUT-1 and GLUT-2) which in turn facilitate myocyte glucose uptake. ${ }^{10}$ Therefore, diffuse myocardial ${ }^{18}$ FDG uptake in the nonfasting state may be a normal physiologic finding. ${ }^{11}$ In some healthy individuals, ${ }^{18}$ FDG uptake may be more heterogeneous, and may be present even in the fasting state. At the same time, inflamed tissue, such as that seen in $\mathrm{CS}$, can also increase glucose utilization ${ }^{12}$ and result in elevated intramyocardial levels of ${ }^{18}$ FDG. The appearance of ${ }^{18}$ FDG uptake in inflamed tissues may be indistinguishable from heterogeneous or focal ${ }^{18} \mathrm{FDG}$ uptake in healthy myocardium, underscoring the importance of suppression of normal physiologic myocardial ${ }^{18}$ FDG uptake. Current means of suppressing physiologic myocardial ${ }^{18}$ FDG uptake for CS PET imaging include (1) prolonged fasting, (2) low-carbohydrate diet (LCD) with or without high fat, ${ }^{13}$ and/or (3) use of intravenous unfractionated heparin (UFH) to stimulate lipolytic activity and increase free fatty acid (FFA) levels. ${ }^{14}$ Limited studies suggest varying degrees of efficacy of these protocols. Similarly, prior studies using other means of physiologic suppression such as shorter fasting protocols have noted varying degrees of myocardial ${ }^{18}$ FDG uptake in a majority of patients, ${ }^{15}$ 
again underscoring the need for additional studies and novel protocols to enhance cardiac ${ }^{18}$ FDG-PET as a tool in CS.

In the current issue of the Journal of Nuclear Cardiology, Manabe and colleagues assessed both the qualitative and quantitative effects of two patient preparation protocols, an 18 -h fast with $\mathrm{LCD}(<5 \mathrm{gm})$ vs a minimum 6-h fast without LCD, on diffuse left ventricular (LV) ${ }^{18}$ FDG-PET uptake and plasma free fatty acid (FFA) levels in patients with suspected CS. Qualitative assessment by two different blinded nuclear medicine physicians demonstrated complete physiologic ${ }^{18}$ FDG suppression in all patients undergoing the 18 -h fast with LCD compared to $72 \%$ of patients who underwent the 6-h fast only, a difference which was statistically significant $(P=.0041)$. Quantitative analysis showed no significant difference in maximal standard uptake value (SUVmax) between the two groups. Furthermore, the authors also noted significantly lower fasting plasma glucose and higher FFA levels in the prolonged fast and LCD group compared to the 6-h fast group. Interestingly, patients with diffuse LV ${ }^{18} \mathrm{FDG}$ uptake showed significantly lower FFA levels than patients without diffuse LV ${ }^{18}$ FDG uptake. Lastly, the current study demonstrated significantly higher FFA levels after vs before UFH administration, but no significant difference in FFA levels between the two preparation groups after UFH administration.

Until now, complete suppression of physiologic ${ }^{18}$ FDG uptake in all patients has not been reported in the literature. The results of this study using combined 18-h fast with LCD are highly promising for enhancing the assessment of CS by cardiac imaging with ${ }^{18}$ FDG-PET. The authors are also congratulated for adding substantially to the literature in this area, which has been limited. Prior studies have mainly addressed the effect of 18-h fasting preparation on suppression of myocardial physiologic FDG uptake. The current study also addresses the utilization of UFH as a means by which to acutely raise FFA and thereby theoretically suppressing myocardial ${ }^{18} \mathrm{FDG}$ uptake. Previous reports have demonstrated that fasting was more effective than UFH in suppressing ${ }^{18} \mathrm{FDG}$ uptake. ${ }^{16}$ In the current study, Manabe and colleagues noted that while UFH does increase FFA, it does not effectively reduce diffuse ${ }^{18} \mathrm{FDG}$ uptake. This is also a crucial finding from the study given the possible risks of UFH administration.

Despite these important findings by Manabe et al, several critical issues remain to be addressed when considering optimizing cardiac ${ }^{18}$ FDG-PET protocols. First, while Manabe and colleagues demonstrated the complete suppression of physiologic myocardial ${ }^{18} \mathrm{FDG}$ uptake, it is unclear whether this finding impacted how CS images were interpreted and the resultant overall accuracy in CS detection by ${ }^{18}$ FDG-PET. Providing evidence of clinical utility is an essential step towards developing universal patient preparation protocols for CS detection. Second, as noted by Manabe et al in the limitations section, the exact fasting times for each group were not available. Recent literature has demonstrated poor compliance with fasting instructions and emphasize the need for further patient education. ${ }^{17,18}$ Poor compliance with dietary instructions may have led to some patients in the 6-h fasting group to actually fast for longer than advised and those in the 18-h group to fast for less than the recommended time ultimately leading to possible contamination of the study groups. Third, it remains unclear whether the prolonged fast or the LCD was the primary factor in suppressing physiologic ${ }^{18}$ FDG uptake or if both were necessary components in achieving complete suppression. If diet is the primary factor, then a prolonged fast could perhaps be avoided. This would be preferable given that one patient in the Manabe study had to be removed due to a low glucose level and given the concerns that a prolonged fast without insulin use (as was the protocol in the current study) might cause untoward complications in patients with insulin-requiring diabetes mellitus. Fourth, the current study spanned over a decade and thereby may have been influenced by changes in practice patterns. For instance, three different PET imaging systems were employed with varied detector crystals and methods of attenuation which could potentially influence image quality and ultimately the interpretation of the presence of ${ }^{18} \mathrm{FDG}$ uptake. Manabe and colleagues cite a meta-analysis by Youssef et al that utilized different PET systems to analyze CS and suggest that the varied operating systems had little impact upon interpretation. ${ }^{7}$ However, it is important to note that the study period of Youssef's work was much shorter (3 years) than the current study and therefore may not have been subjected to as many protocol changes and advancements in technology as the current study. Fifth, as protocols become further refined it will be important to remember that other non-pathologic (papillary muscles, crista terminalis, lipomatous hypertrophy of the left atrial septum) and pathologic (myocarditis and left ventricular hypertrophy) phenomena will impact the pattern of ${ }^{18} \mathrm{FDG}$ uptake regardless of the preparation protocol used. In the current study patients with coronary artery disease, myocarditis, cardiomyopathy, and valvular heart disease were excluded. These are important considerations when deciding whether or not a protocol has widespread clinical applicability. Furthermore, recent literature advocates the use of a high-fat diet in conjunction with LCD for suppression of myocardial ${ }^{18} \mathrm{FDG}$ uptake, ${ }^{13,19,20}$ a protocol recommended and utilized by many experts. The current study does not incorporate a 
high-fat diet with LCD protocol, which should be included in future studies to examine its effectiveness in suppressing myocardial ${ }^{18}$ FDG uptake both qualitatively and quantitatively. Lastly, experts advocate the use of ${ }^{13} \mathrm{~N}$-ammonia in conjunction with cardiac ${ }^{18}$ FDG-PET imaging for CS evaluation ${ }^{21}$ but the former is yet to be fully investigated to determine its diagnostic accuracy in the CS population.

Given the recent incorporation of cardiac ${ }^{18}$ FDGPET into the Heart Rhythm Society guidelines for the assessment of $\mathrm{CS}^{8}{ }^{8}$ it is imperative that standardized protocols be developed for cardiac ${ }^{18}$ FDG-PET imaging for CS. The findings by Manabe and colleagues represent a significant contribution towards the ultimate goal of creating a universal ${ }^{18}$ FDG-PET preparation protocol for CS and is a step in the right direction of optimizing and standardizing PET imaging for the diagnosis and management of CS.

\section{Disclosures}

The authors have nothing to disclose.

\section{References}

1. Silverman KJ, Hutchins GM, Bulkey BH. Cardiac sarcoid: A clinicopathologic study of 84 unselected patietns with systemic sarcoidosis. Circulation 1978;58:1204-11.

2. Doughan AR, Williams BR. Cardiac sarcoidosis. Heart 2006;92: 282-8.

3. Yazaki Y, Isobe M, Hiroe M, et al. Prognostic determinants of long-term survival in Japanese patients with cardiac sarcoidosis treated with prednisone. Am J Cardiol 2001;88:1006-10.

4. Cooper LT, Baughman K, Feldman AM, et al. The role of endomyocardial biopsy in the management of cardiovascular disease. Circulation 2007;1216:2216-33.

5. Mañá J, Gámez C. Molecular imaging in sarcoidosis. Curr Opin Pulm Med 2011;17:325-31.

6. Ohira H, Tsujino I, Yoshinaga K, et al. 18F-fluoro-2-deoxyglucose positron emission tomography in cardiac sarcoidosis. Eur J Nucl Med Mol Imaging 2011;38:1773-83.

7. Youssef G, Leung E, Mylonas I, et al. The use of 18F-FDG PET in the diagnosis of cardiac sarcoidosis: A systemic review and metaanalysis including the Ontario experience. J Nucl Med 2012; $52: 241-8$.
8. Birnie DH, Sauer WH, Bogun F, et al. HRS expert consensus statement on the diagnosis and management of arrythmias associated with cardiac sarcoidosis. Heart Rhythm 2014;11:1304-23.

9. Manabe O, Ohira H, Yoshinaga K, et al. Elevated 18F-flurodexoyglucose uptake in the interventricular septum is associated with atrioventricular block in patients with suspected cardiac involvement sarcoidosis. Eur J Nucl Med Mol Imaging 2013;40:1558-66.

10. Maurer AH, Burshteyn M, Adler LP, Steiner RM. How to differentiate benign versus malignant cardiac and paracardiac $18 \mathrm{~F}$ FDG uptake at oncologic PET/CT. RadioGraphics 2011;31:1287305.

11. Ishida Y, Yoshinaga K, Miyagawa M, et al. Recommendations for F-Flurodeoxyglucose positron emission tomography imaging for cardiac sarcoidosis: Japanese Society of Nuclear Cardiology Reccomendations. Ann Nucl Med 2014;28:393-403.

12. Newsholme P, Newsholme EA. Rates of utilization of glucose, glutamine and oleate and formation of end-products by mouse peritoneal macro. Biochem J 1989;261:211-8.

13. Williams G, Kolodny G. Suppression of myocardial 18F-FDG uptake by preparing patients with high-fat, low-carbohydrate diet. Am J Roentgenol 2008;190:W151-6.

14. Ito K, Morooka M, Okazaki O. Efficacy of heparin loading during an 18F-FDG PET/CT examination to search for cardiac sarcoidosis activity. Clin Nucl Med 2013;38:128-30.

15. Maurer AH, Burshteyn M, Adler LP, Gaughan JP, Steiner RM. Variable cardiac 18 FDG patterns seen in oncologic positron emission tomography computed tomography importance for differentiating normal physiology from cardiac and paracardiac disease. J Thorac Imaging 2012;27:263-8.

16. Morooka M, Moroi M, Ito K, et al. Heparin vs. long fasting method: Which inhibits the FDG myocardial physiological uptake more strongly? J Nucl Med 2013;54:406.

17. Kyrtatos PG, Constandinou N, Loizides S, Mumtaz T. Improved patient education facilitates adherence to preoperative fasting guidelines. J Perioper Pract 2014;24:228-31.

18. Nadella V, Gupta M, Brown J, et al. Evaluation of adherence to recommended fasting guidelines in pediatric surgery in a teaching hospital in the UK: 10AP3-3. Eur J Anaesthesiol 2013;30:162-3.

19. Cheng VY, Slomka P, Ahlen M, et al. Impact of carbohydrate restriction with and without fatty acid loading on myocardial $18 \mathrm{~F}-$ FDG uptake during PET: A randomized controlled trial. J Nucl Cardiol 2010;17:286-91.

20. Kobayashi Y, Kumita S, Fukushima Y. Significant suppression of myocardial $18 \mathrm{~F}$-fluorodeoxyglucose uptake using 24-h carbohydrate restriction and a low-carbohydrate, high-fat diet. J Cardiol 2013;62:314-9.

21. Yamagishi H, Shirai N, Takagi M, et al. Identification of cardiac sarcoidosis with 12N-NH 3/18F-FDG PET. J Nucl Med 2003; 44:1030-6. 